\title{
The Challenges of Making Care Safer: Leadership and System Transformation
}

\author{
G. Ross Baker
}

T Ten years ago, in September 2002, the National Steering Committee on Patient Safety delivered its report urging the development of the Canadian Patient Safety Institute and enhanced efforts to identify and reduce the risk of patient harm across the healthcare system. Two years later, the Canadian Adverse Events Study (Baker et al. 2004) provided data on patient safety in acute care - data that reported levels of harm far greater than most suspected. Today, virtually all Canadian healthcare organizations have goals around improving the safety and quality of care, and many have implemented reporting systems that identify patient safety incidents and track the implementation of recommendations to reduce hazards. In only a decade, patient safety has been transformed from the esoteric interest of a small number of champions to an essential component of healthcare performance across Canada. Today, patient safety is a fundamental prerequisite for the healthcare system: quality is impossible unless patients are protected from unintended harm.

Yet despite clear goals and considerable investments to improve patient safety, the gains have been limited. Healthcare-associated infections remain a major source of patient morbidity and mortality (Umscheid et al. 2011); adverse drug events continue to occur in hospitals (Classen 2010) and many other settings (e.g., Thomsen et al. 2007); and many other risks, such as pressure ulcers and patient falls, resist efforts to reduce their incidence. Although the problems are now well known, progress is slow. One study of a sample of hospitals in one US state showed little evidence of improvement over 10 years (Landrigan et al. 2010).
Even more troubling are the challenges of creating effective solutions. As Fernandes and Shojania (2012) report in this special issue, medication reconciliation offers clear benefits when implemented in a robust and effective fashion; but many organizations have created only a superficial and ineffective process. Efforts to improve hand hygiene gain traction in many settings, only to have compliance return to low levels when attention moves elsewhere. And the problems span organizational boundaries. Dhalla and colleagues (2012) explore the reasons why many patients experience adverse events following hospital discharge. The handoff between the hospital and the community fails due to inadequate plans or poor communication about follow-up visits or medications. Safe and effective care requires careful coordination between caregivers in multiple settings and quick, informed decisions based on new knowledge about a patient's condition. As a result, the opportunities for failure are frequent. Patient safety in the home presents additional challenges: as Stevenson and her colleagues detail (2012), every home environment is different and the resources available to identify and respond to risks are limited.

High reliability in many industries comes from automating complex processes; however, healthcare remains a largely human endeavour - which is mostly a good thing. But humans are fallible, systems are imperfect and patient safety is elusive.

Still some progress is apparent. One of the important benefits of the emphasis on patient safety and quality improvement in the past decade has been the development of new approaches to clinical improvement. Patient safety "campaigns" beginning 
with the Institute for Healthcare Improvement 100,000 Lives Campaign and the Canadian Safer Healthcare Now! initiative have mobilized large-scale efforts and motivated greater engagement. While notable themselves for spurring action, often with defined timetables and goals, these initiatives incorporated several important and innovative features. First, they were designed around the Model for Improvement, a simple, effective strategy for setting aims, selecting measures and smallscale changes and then testing these changes in local settings (Langley et al. 2009). Although the logic for the Model for Improvement is rooted in a long tradition, many teams and organizations first used it as part of Safer Healthcare Now! and similar patient safety initiatives. Second, these campaigns incorporated an effective knowledge translation strategy that translated evidence around effective practice into "bundles" of specific recommended practices. In the bundle for central line care, for example, teams were asked to carry out five practices: hand hygiene; maximal barrier precautions; chlorhexidine skin antisepsis; optimal catheter-site selection, avoiding the femoral vein for central venous access in adult patients; and daily review of the necessity of central lines, with unnecessary lines promptly removed. Although there was evidence for the use of each of these elements, their prominent inclusion in a list of key practices and the highlighted experience of teams that were able to reduce central line infections using these practices provided considerable traction for local leaders who sought to engage teams in improving care.

The identification of effective practices and their "translation" into bundles has linked broader patient safety goals into daily clinical practice. But at the same time, the variation in the success of different teams in adopting these practices makes it evident that knowledge of effective practice alone is insufficient to improve performance. Improved patient safety relies not just on knowledge of safe practices but also on creating care environments that support individuals and teams to identify, adapt and spread these practices. In the Keystone project, which involved clinicians from 103 intensive care units (ICUs) in 55 Michigan hospitals in reducing the rate of catheter-related bloodstream infections (CR-BSIs; Pronovost et al. 2006), the evidence of effective practices was linked to a strategy to engage, educate and execute these practices in local settings and to evaluate their impact. Rather than assuming that knowledge of "what works" was sufficient, the project explicitly linked the new practices to efforts to improve local unit safety cultures and to engage organizational leaders in reviewing and supporting unit-based change. Borrowing from Ronald Heifitz's useful distinction between "technical" changes based on scientific evidence and "adaptive" changes involving attitudes, values and culture (Heifitz 1994), Pronovost and colleagues (2006) recognized that the alterations in ICU practice incorporated in their efforts to reduce CR-BSIs were more than just changes in simple practice. These changes had to be rooted in the critical care unit culture and supported by leadership at the unit and organizational levels.

Implementing effective practices into daily routines is more than simply acknowledging culture; the dynamics of change are variable and complex. Some units absorb new tools and approaches such as the central line infection bundle or safe surgery checklist; others see these ideas as a challenge to existing routines and relationships. While patient safety practices have become accreditation requirements and publicly reported performance measures, these requirements are insufficient to overcome resistance in many settings - they can create superficial compliance rather than reliable practice.

Rosabeth Moss Kanter noted that "everything can look like a failure in the middle" (1999: 20). Sound ideas for improving patient safety and well-conceived plans to implement these changes may start enthusiastically, but they inevitably hit a plateau. Leaders must sell their ideas and engage staff, not expect blind obedience. Healthcare organizations are slow to change because they are complex professional bureaucracies and patterns of local work become deeply ingrained. Scepticism about change is rooted not only in a defence of professional autonomy but also in a need to assess whether new ideas are effective or possibly harmful. Over the past decade, there has been a growing recognition of the need to identify risks and create strategies to prevent or mitigate the potential harm that results from these risks. The challenge for the next decade will be to create integrated and sustainable strategies to incorporate safe practices and safety strategies into Canadian healthcare organizations. A small number of key elements will help to determine the effectiveness of these efforts.

\section{Strategic Elements for a Safer System}

Government budgets and recent reports, such as the Drummond report (2012) in Ontario, herald a new era of cost constraints in healthcare. These may undermine current efforts to implement patient safety practices; at a minimum, they will force organizations to take a harder look at their current investments in quality improvement and patient safety. While patient safety concerns have been a high priority for the past decade, moving forward, the realistic prospect is that patient safety advocates will need to be more disciplined in arguing the "business case" for safety. Poor-quality care is typically more expensive than good-quality care. But capturing the savings from new patient safety practices is often difficult. The ICU patient safety initiatives of Peter Pronovost and his colleagues translated into both a substantial improvement in care and large cost savings (Waters et al. 2011). Prioritizing efforts that offer considerable savings is an obvious strategy for preserving current investments in quality and patient safety staff and other resources. Rather than see cost containment and patient safety as competing goals, we should welcome the opportunity to reduce harm while limiting or reducing costs. An additional challenge is that Canadian hospitals are funded 
largely through global budgets. Individual organizations may not garner savings from reductions in adverse events since their beds remain filled with other patients - so the challenge will be to reap those savings on a broader system level while maintaining a commitment to improvement at the local level.

Too many patient safety initiatives have had limited scope and, thus, minimal impact. But if the goal is to reduce harm and costs by focusing on high-frequency or high-cost events, patient safety interventions have to scale up. Sometimes the limits on scale stem from variable enthusiasm: for example, as Chris Hayes (2012) notes in this volume, some surgeons have adopted the surgical checklist while others avoid it. But if errors can happen to any surgeon in any operation, then ensuring widespread adoption is key to reducing harm and conserving resources. Testing new ideas and adapting improvements to local practice environments make sense given the differences between Toronto and Timmins, or Calgary and Cape Breton. However, when interventions prove effective, leaders must require commitment from those providing care. The test of effective patient safety is no longer success on individual units but, rather, the creation of reliable and safe care across large systems.

In many Canadian hospitals, the responsibility for clinical care is delegated to program or unit directors and clinical leads; but if patient safety and quality of care are truly strategic goals, they need to be embraced by senior leaders, with clear accountability from the board to the ward. New legislation in Ontario, the Excellent Care for All Act, has clarified the responsibilities of boards and senior leadership for quality of care; and the publication of quality improvement plans by all Ontario hospitals emphasizes both current levels of performance and the scale of hospitals' quality goals. Yet, setting goals is only the beginning of the challenge. Aligning activities and investments on quality improvement strategies across executive portfolios can be difficult, as is creating a portfolio of projects that can address the quality goals. Recent work by Trillium Health Care in Ontario demonstrates how driver diagrams and other management tools have helped to focus and align patient safety and quality activities in that organization (Cochrane et al. 2011).

Capacity and capability for change is another important issue. Helen Bevan identified capacity as "having the right number and level of people who are actively engaged and able to take action" and capability "means that those people have the confidence and the knowledge and skills to lead the change"(2010: 2). The growing pressures of daily work create a disincentive for staff to engage in improvement, and organizations faced with slow and uncertain improvement often employ external change agents to speed these efforts. However, while change consultants can help to start change, it is the ongoing capability of organizations to develop new ways of working that is key to enduring improvements (Parcell and Collison 2009, as cited in Bevan 2010). Canadian healthcare organizations have relatively few staff with expertise in quality improvement methods and patient safety. These staff are often burdened with multiple responsibilities and ambitious programs of work. Since the pace of change is limited by the capabilities of front-line units, supported by quality improvement experts, to make improvements, the pace is usually slow. Experience in a number of highperforming healthcare organizations, such as Intermountain Healthcare and the Henry Ford Health System in the United States and Jönköping County Council in Sweden, demonstrates that greater investment in this capability accelerates the pace of improvement (Baker et al. 2008; Baker and Denis 2011a).

Physician engagement is a particularly critical issue. No substantial change in clinical care is possible without the full involvement of physicians. Efforts to transform healthcare systems - improving safety and quality while limiting increases in costs - must engage informed physicians as part of the team. Yet this has been difficult in many settings. There are encouraging efforts in Saskatchewan and elsewhere in Canada to develop a physician cohort with expert quality improvement skills, but these initiatives need to be broadened. Leaders in healthcare organizations must recognize that developing the skills of a handful of physicians to assume leadership roles is no longer sufficient. Rather, leaders need to create a distributed network of physician champions as part of medical leadership strategies that facilitate broad-scale improvement among their physician colleagues (Baker and Denis 2011b). These physicians (and other clinicians) will become role models. Their insights into clinical processes linked with knowledge of quality improvement will provide the mechanisms for identifying and implementing sustainable quality gains.

Achieving higher-quality and safer care without increasing long-term costs requires the redesign of existing systems of care. Improvement starts with identifying waste and focusing on value. The current and growing interest in applying Lean thinking and Lean production ideas to healthcare stems from this need. Lean initiatives provide the tools to systematically examine work processes, identify improvements and implement new systems and environments. New programs, such as Releasing Time to Care: The Productive Ward, in Saskatchewan and Ontario offer systematic ways to examine and improve nursing care and patient unit environments, with the potential to improve efficiency, increase patient safety and quality, boost staff satisfaction and enhance the patient experience. Yet working on large-scale improvement is an enormous task, and the rate-limiting step is our ability to create capability - the skill, energy and insight- at the front lines to improve care.

In a time of restraint, it might seem ambitious, or even foolhardy, to suggest that we need a broad investment in improvement skills in leaders, clinicians and managers. But this is exactly what the system needs. Rather than cutting education and support (as is usually done in times of restraint), we should 
be investing in programs to build skills, provide resources to seek out and test innovative new ideas, and create the energy that helps to mobilize staff across the system. Large-scale changes, in healthcare or elsewhere, require mobilizing commitment, not just restructuring of pieces and programs (Bate et al. 2004; NHS Institute for Innovation and Improvement n.d.). Reports over the past 15 years have highlighted the critical issues facing the Canadian healthcare system. These issues remain, and recent reports, such as the Drummond report (2012), have reinforced their urgency. But there is still no detailed plan in most of Canada for how to execute the changes needed to improve safety and quality at a large scale across the continuum of care with the currently available resources. The costs of developing leadership skills and improvement knowledge and of investing in staff and innovation to achieve a sustainable healthcare system are relatively modest. The achievable outcomes that would come from such efforts are likely the only bridge from the current system - which wastes resources and breeds frustration in patients, staff and leadership alike - to a sustainable, accessible and safe healthcare system. $\mathrm{HQ}$

\section{References}

Baker, G.R., A. MacIntosh-Murray, C. Porcellato, L. Dionne, K. Stelmacovich and K. Born. 2008. High Performing Healthcare Systems: Delivering Quality by Design. Toronto, ON: Longwoods Publishing Corporation.

Baker, G.R. and J.L. Denis. 2011a. A Comparative Study of Three Transformative Healthcare Systems: Lessons for Canada. Ottawa, ON: Canadian Health Services Research Foundation.

Baker, G.R. and J.L. Denis. 2011b. "Medical Leadership in Health Care Systems: From Professional Authority to Organizational Leadership." Public Money and Management 31(5): 355-62.

Baker, G.R., P.G. Norton, V. Flintoft, R. Blais, A. Brown, J. Cox et al. 2004. "The Canadian Adverse Events Study: The Incidence of Adverse Events among Hospital Patients in Canada." Canadian Medical Association Journal 170(11): 1678-86.

Bate, P., G. Robert and H. Bevan. 2004. "The Next Phase of Healthcare Improvement: What Can We Learn from Social Movements?” Quality and Safety in Health Care 13: 62-66.

Bevan, H. 2010. "How Can We Build Skills to Transform the Health Care System?” Journal of Research in Nursing 15(2): 139-48.

Classen, D.C., L. Jaser and D.S. Budnitz . 2010. "Adverse Drug Events among Medicare Patients: Epidemiology and National Estimates from a New Approach to Surveillance." Joint Commission Journal on Quality and Patient Safety 36(1): 12-19.

Cochrane, P., A. Ginsburg, G. Spencer and F. Marani. 2011. "Uniting Board to Bedside: The Use of Driver Diagrams in Quality Monitoring and Improvement at Trillium Health Centre." Healthcare Quarterly 14(4): 54-59.

Drummond, D. and the Commission on the Reform of Ontario's Public Services. 2012. Public Services for Ontarians: A Path to Sustainability and Excellence. Toronto, ON: Queen's Printer for Ontario. Retrieved February 27, 2012. <http://www.fin.gov.on.ca/en/reformcommission/ chapters/report.pdf>.

Dhalla, I.A., T. O’Brien, F. Ko and A. Laupacis. 2012. “Toward Safer
Transitions: How Can We Reduce Post-Discharge Adverse Events?" Healthcare Quarterly 15(Special Issue): 63-67.

Fernandes, O. and K.G. Shojania. 2012. "Medication Reconciliation in the Hospital." Healthcare Quarterly 15(Special Issue): 42-49.

Hayes, C. 2012. "The Surgical Safety Checklist: Heightened Patient Safety through Improved Teamwork." Healthcare Quarterly 15(Special Issue): $57-62$.

Heifitz, R. 1994. Leadership without Easy Answers. Cambridge, MA: Harvard University Press.

Kanter, R.M. 1999. "The Enduring Skills of Change Leaders.” Leader to Leader 13: 15-22.

Landrigan C.P,. G.J. Parry, C.B. Bones, A.D. Hackbarth, D.A. Goldmann and P.J. Sharek. 2010. "Temporal Trends in Rates of Patient Harm Resulting from Medical Care." New England Journal of Medicine 363: 2124-34.

Langley, G.J., R.D. Moen, K.M. Nolan, T.W. Nolan, C.L. Norman and L.P. Provost. 2009. The Improvement Guide (2nd ed.). San Francisco, CA: Jossey-Bass.

National Steering Committee on Patient Safety. 2002. Building a Safer System: A National Integrated Strategy for Improving Patient Safety in Canadian Health Care. Ottawa, ON: Author. Retrieved February 27, 2012. <http://rcpsc.medical.org/publications/building_a_safer_ system_e.pdf $>$.

NHS Institute for Innovation and Improvement. n.d. Helen Bevan on Social Movement Thinking: A Set of Ideas Whose Time Has Come? Coventry, United Kingdom: Author. Retrieved February 14, 2012. $<$ http://www.institute.nhs.uk/quality_and_value/introduction/helen_ bevan_on_social_movement_thinking\%3A_a_set_of_ideas_whose_ time_has_come?.html>.

Pronovost, P.J., D.M. Needham, S. Berenholtz, D. Sinopoli, H. Chu, S. Cosgrove et al. 2006. "An Intervention to Decrease Catheter-Related Bloodstream Infections in the ICU." New England Journal of Medicine 355(26): 2725-32.

Stevenson, L., A. Lang, M. Macdonald, J. Archer and C. Berlanda. 2012. "Safety in Home Care: Thinking Outside the Hospital Box." Healthcare Quarterly 15(Special Issue): 68-72.

Thomsen L.A., A.G. Winterstein, B. Sandergarrd L.S. Haugbelle and A. Melander. 2007. "Systematic Review of the Incidence and Characteristics of Preventable Adverse Drug Events in Ambulatory Care." The Annals of Pharmacotherapy 41(9): 1411-26.

Umscheid C.A,. M.D. Mitchell J.A. Doshi et al. 2011. "Estimating the Proportion of Healthcare-associated Infections that are Reasonably Preventable and the Related Mortality and Costs." Infection Control and Hospital Epidemiology 32(2): 101-14

Waters, H.R., R. Korn, E. Colantuoni, S.M. Berenhotz, C.A. Goeschel, D.M. Needham et al. 2011. "The Business Case for Quality: Economic Analysis of the Michigan Keystone Patient Safety Program in ICUs." American Journal of Medical Quality 26(5): 333-39.

\section{About the Authors}

G. Ross Baker, PhD, a professor in the Institute of Health Policy, Management and Evaluation at the University of Toronto Ross chaired an Advisory Panel on Avoidable Hospitalizations for the Ontario Ministry of Health and Long Term Care whose report was released by the Ministry in November 2011. He also served as a member of the King's Fund (London, UK) Commission on Leadership and Management in the NHS in 2010-2011. 